\section{Physical and Dosimetric Aspect of Euromechanics Add-on Multileaf Collimator on Varian Clinac 2100 C/D}

\author{
Rohani S. A. ${ }^{1}$, Mahdavi S. R. ${ }^{2}$, Mostaar A. ${ }^{3}$, Ueltzhöffer S. ${ }^{4}$, \\ Mohammadi R. ${ }^{5}$, Geraily Gh. ${ }^{1 *}$
}

\section{ABSTRACT}

Background: Before treatment planning and dose delivery, quality assurance of multi-leaf collimator (MLC) has an important role in intensity-modulated radiation therapy (IMRT) due to the creation of multiple segments from optimization process.

Objective: The purpose of this study is to assess the quality control of MLC leaves using EBT3 Gafchromic films.

Material and Methods: Leaf Position accuracy and leaf gap reproducibility were checked with Garden fence test. The garden fence test consists of 5 thin bands A) $0.2 \mathrm{Cm}$ width spaced at $2 \mathrm{Cm}$ intervals and B) $1 \mathrm{Cm}$ width spaced at $1 \mathrm{Cm}$ intervals. Each leaf accuracy was analyzed with measuring the full-width half-maximum (FWHM). Maximum and average leaf transmission were measured with gafchromic EBT3 films from Ashland for both $6 \mathrm{MV}$ and $18 \mathrm{MV}$ beams.

Results: Leaf positions were found to be in a range between $1.78-2.53 \mathrm{~mm}$, instead of nominal $2 \mathrm{~mm}$ for the test $\mathrm{A}$ and between $9.09-10.36 \mathrm{~mm}$, instead of nominal $10 \mathrm{~mm}$ for the test $\mathrm{B}$. The Average radiation transmission of the MLC was noted $1.79 \%$ and $1.98 \%$ of the open $10 \times 10 \mathrm{Cm}^{2}$ field at isocenter for $6 \mathrm{MV}$ and 18 MV beams, respectively. Maximum radiation transmission was noted $4.1 \%$ and $4.4 \%$ for $6 \mathrm{MV}$ and $18 \mathrm{MV}$ beams, respectively.

Conclusion: In this study, application of gafchromic EBT3 films for the quality assurance of Euromechanics multileaf collimator was studied. Our results showed that the average leaf leakage and positional accuracy of this type of MLC were in the acceptance level based on the Protocols.

\section{Keywords}

Multileaf Collimator, Mechanical Test, Garden Fence Test, Leaf Transmission, Leaf End Transmission

\section{Introduction}

$\mathrm{B}$ efore treatment planning and dose delivery, quality assurance of multi-leaf collimator (MLC) has an important role in intensitymodulated radiation therapy (IMRT) due to the creation of multiple segments from optimization process. Transmission from the MLC should be considered completely when using MLC for the IMRT technique [1-3]. Transmission increases the dose in and out of target volume which is not desirable.

There are two inaccuracies in leaf-positioning, including random and systematic ones. The random leaf-positioning inaccuracies effects
${ }^{1}$ Department of Medical Physics, Tehran Univer-

sity of Medical Sciences,

Tehran, Iran

${ }^{2}$ Radiation biology re-

search center \& medical

Physics department,

faculty of medicine, Iran

University of Medical Sci-

ences, Tehran, Iran

${ }^{3}$ Department of Medical

Physics, School of Medi-

cine, Shahid Beheshti

University of Medical

Sciences, Tehran, Iran

${ }^{4}$ Department of Clinic

for Radiotherapy and

RadioOncology, Medical

Faculty Mannheim of the

University of Heidelberg,

Heidelberg, Germany

${ }^{5}$ Department of Medical

Physics, Iran University

of Medical Sciences,

Tehran, Iran

*Corresponding author: Gh. Geraily

Department of Medical Physics, Tehran University of Medical Science, Tehran, Iran

E-mail: gh-geraily@sina. tums.ac.ir

Received: 4 November 2018 Accepted: 20 November 2018 
is stochastic, moreover, after some treatment fraction, they will be reduced. The effect of systematic leaf-positioning inaccuracies will Cause large dose deviations inside the target volume during treatment of a patient who has been planned by treatment planning system. One of the reasons caused the inaccuracy in leaf-positioning is wrong calibration of leaves. Amount of the wrong calibration can be detected with leaf-positioning tests $[4,5]$.

Several types of MLCs are commercially available. Some of their properties are different from conventional collimators, including design, physical and dosimetric characteristics. MLCs have been used to conform the field of treatment to the target volume for several years. Several studies have investigated the dosimetric characteristic of some MLCs used for static fields [6-8]. Several studies have investigated dosimetric properties of MLC systems using small ionization chambers and radiographic films [9, 10], They reported some characteristics such as leaf transmission, leakage and beam penumbra measurements. Small ionization chambers have the limitation due to their finite size and effective active area (EAA), They measure field sizes larger than their EAA precisely, but for field sizes smaller than their EAA, partial volume effects might have an impact on their response. On the other hand, although the advantages of the radiographic films are high spatial resolution and energy independency, they might show an overestimation of beam penumbra due to their over-response to scattered photons with low energy.

In this study, we used EBT3 films which are the newest version of the gafchromic EBT series. The EBT3 is an advanced version of the EBT2 film [11]. One of the advantages of the EBT3 rather than EBT2, is the film scan orientation independency [12]. This is the first installation of 60-leaf PMLC from Euromechanics company and the aim of this study is to assess the quality control of MLC by using the EBT3 gafchromic films.

\section{Material and Methods}

\section{A. MLC Description}

The Euromechanics PMLC (Euromechanics Medical GmbH, Germany) consists of 60 leaves, each leaf is an independently motor driven and has an independent position measuring system. These 60 leaves are positioned in 2 banks and have 50\% over-traveling. The MLC leaves are $63 \mathrm{~mm}$ thick and made of high-density tungsten alloy, they are single focus with rounded front end with tongue and grooves design. The physical width for all leaves are $4.3 \mathrm{~mm}$, and project width of around $7 \mathrm{~mm}$ at the isocenter depends on the clearance of different LINAC Types.

\section{B. EBT3 Gafchromic Films}

The gafchromic EBT3 (Ashland Specialty Ingredients, NJ, USA) is designed for the measurement of absorbed doses of ionizing radiation and appropriate for high energy photons and available in sheets of $8 * 10$ inch. According to the manufacturer specification, it consists of an active layer with approximate thickness $28 \mu \mathrm{m}$ coated on both sides by a protective $125 \mu \mathrm{m}$ polyester layer (Figure 1). For these experiments, the films with Lot No. 09071602 were used. Film dosimetry procedures were done based on recommendations of the manufacturer [13] and AAPM TG-55 protocol [14].

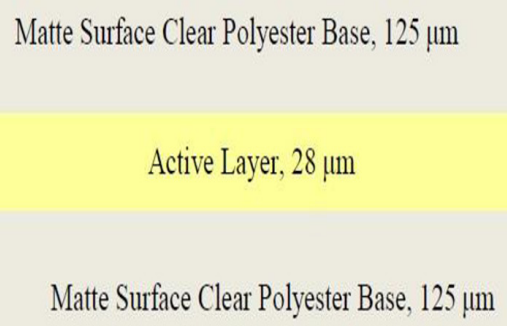

Figure 1: Structure of Gafchromic EBT3 Dosimetry Film. 
C. Mechanical Test (Leaf Position Accuracy)

The leaf position accuracy and leaf gap reproducibility were checked with Garden fence test. The garden fence test consists of 5 thin bands A) $0.2 \mathrm{Cm}$ width spaced at $2 \mathrm{Cm}$ intervals and B) $1 \mathrm{Cm}$ width spaced at $1 \mathrm{Cm}$ intervals. Each leaf accuracy and deviations from planned leaf positions were analyzed with measuring the full-width half-maximum (FWHM) (Figure 2) [15-19]. This fields were irradiated on EBT3 Gafchromic film at SSD $100 \mathrm{Cm}$ placed on the treatment couch without any buildup to have sharper peaks.

(A)
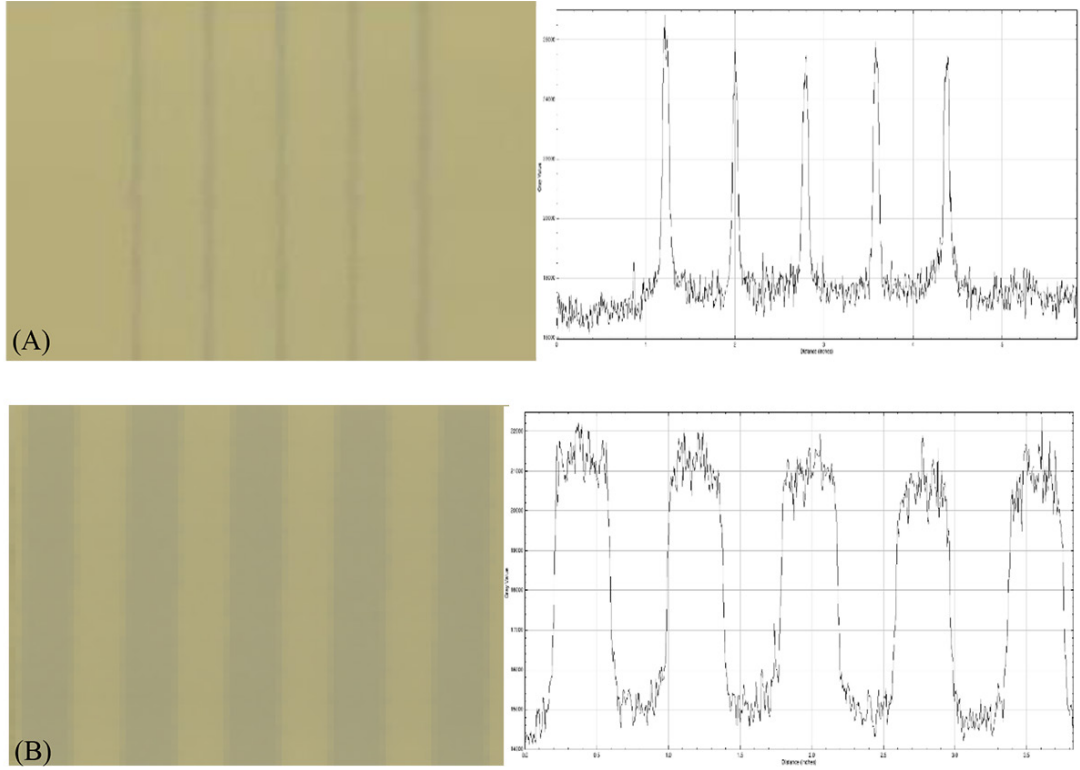

Figure 2: Exposed EBT3 film and plotted profiles of 5 band in SSD=100 $\mathrm{Cm}$ for Garden Fence Test, A) $0.2 \mathrm{Cm}$ width spaced at $2 \mathrm{Cm}$ intervals, B) $1 \mathrm{Cm}$ width spaced at $1 \mathrm{Cm}$ intervals.

\section{MLC Transmission}

Leaf transmission is the sum of mid-leaf and inter-leaf transmission. Maximum and average transmission were measured with gafchromic EBT3 Films from Ashland for both $6 \mathrm{MV}$ and $18 \mathrm{MV} \mathrm{X}$ rays. To find the film response to the radiation, 13 pieces of films $\left(5 \times 5 \mathrm{Cm}^{2}\right)$ were located in $3 \mathrm{Cm}$ polyester and SSD $97 \mathrm{Cm}$ and were exposed from 0 to $1300 \mathrm{cGy}$. For measuring the leaf transmission and transmission at the end of the banks (leaf end Transmission), the films were located at SSD 98.5 $\mathrm{Cm}$ and depth of $1.5 \mathrm{Cm}$ for $6 \mathrm{MV}$ Photons and SSD $96.5 \mathrm{Cm}$ and depth of $3.5 \mathrm{Cm}$ for $18 \mathrm{MV}$ Photons, the leaves were completely closed and radiated with $1000 \mathrm{MU}$ for each energy (Figure 3). For measuring the leakage between leaves from opposite banks, the same set up were executed and the leaves were in

\section{Interdigit position.}

All the exposed films and one of un-exposed film were scanned in 48 hours with Microtek scanner model ScanMaker 9800XL Plus (Microtek International, Inc. MRS-3200A3L, China), in three colors (48 bit RGB) with 150 dpi in transmission mode and saved as a tiff format. The .tiff files were analyzed in ImageJ Software (National Institute of Health, Bethesda, MD).

Net optical densities (Net OD) and the standard deviations $(\sigma)$ were determined according to the equations (1-2) $[12,20,21]$ :

$$
\begin{aligned}
& N e t O D=\log \frac{I_{\circ}}{I} \\
& \sigma_{\text {netOD }}=\frac{1}{\operatorname{Ln} 10} \frac{\sigma_{I}}{I}
\end{aligned}
$$

Where $\mathrm{I}_{0}$ and $\mathrm{I}$ are intensities measured for 
Figure 3: Exposed EBT3 film with 1000 MU. A: Scanning for measuring the leaf leakage, B: scanning for measuring the end leaf leakage.

un-irradiated films and irradiated films, respectively.

The Net OD values were imported in MATLAB and to retrieve a dose from net OD Value, the best curve was fitted to this OD values according to Devic proposed function (equation 3) [22]. Levenberg-Marquardt algorithm was used for optimization.

$D_{f i t}=b^{*} N e t O D+C * N e t O D^{n}$

Where $\mathrm{b}$ and $\mathrm{c}$ are the fitting parameters and $\mathrm{n}$ is responsible for the non-linear saturation process of the film at high doses.

Film dosimetry is accompanied with several sources of uncertainties. There are two main sources of uncertainty, Experimental and fitting, the total uncertainty is the root square of the experimental and fitting uncertainty summation [23]. The estimated experimental uncertainty was calculated as below (equation 4) [22]:

$\sigma_{D_{\text {exp }}}(\%)=\frac{\sqrt{\left(b+n \cdot c \cdot n e t O D^{n-1}\right)^{2} \cdot \sigma_{n e t O D}^{2}}}{D_{f i t}} \cdot 100(4)$

Where $\sigma_{\text {NetOD }}$ is optical densities uncertainty which is defined in equation (2). The fitting uncertainty $\left(\sigma_{\text {Dfit }}\right)$ was calculated as below (equation 5):

$\sigma_{D_{f i t}}(\%)=\frac{\sqrt{\left(n e t O D^{2} \cdot \sigma_{b}^{2}+n e t O D^{2 n} \cdot \sigma_{c}^{2}\right)}}{D_{f i t}} \cdot 100(5)$

Where $\sigma_{b}$ and $\sigma_{c}$ are the fitting parameter uncertainties. Finally, the total dose uncertainty was calculated through the following equation (equation 6):

$$
\sigma_{D_{t o t}}(\%)=\frac{\sqrt{\left(n e t O D^{2} \cdot \sigma_{b}{ }^{2}+n e t O D^{2 n} \cdot \sigma_{c}{ }^{2}\right)+\left(b+n \cdot c \cdot n e t O D^{n-1}\right)^{2} \cdot \sigma_{n e t O D}}}{D_{f i t}} \cdot 100
$$

\section{Result and Discussion}

\section{A. Mechanical Test (Leaf Position Accuracy):}

Table 1 shows the results of the garden fence test for the leaf position accuracy for each peak. The leaf positions were found to be in the range between $1.78-2.53 \mathrm{~mm}$, instead of nominal $2 \mathrm{~mm}$ and the average of $2.24 \mathrm{~mm}$ which correspond to a disposition of $0.24 \mathrm{~mm}$ 
Table 1: Results of the measured distance in each Peak in $\mathrm{mm}$ for Garden Fence Test, A) 2mm Bands (Top), B) $10 \mathrm{~mm}$ Bands (Below).

\section{$2 \mathrm{~mm}$ bands}

\begin{tabular}{ccc}
\hline & Mean & SD \\
\hline Peak1 & 2.69 & 0.19 \\
\hline Peak2 & 2.09 & 0.20 \\
\hline Peak3 & 2.13 & 0.22 \\
\hline Peak4 & 2.19 & 0.27 \\
\hline Peak5 & 2.11 & 0.25 \\
\hline Mean & 2.24 & 0.22 \\
\hline & 10 mm bands & \\
\hline & Mean & SD \\
\hline Peak1 & 9.32 & 0.29 \\
\hline Peak2 & 10.13 & 0.17 \\
\hline Peak4 & 9.78 & 0.36 \\
\hline Peak5 & 9.84 & 0.28 \\
\hline Mean & 9.75 & 0.25 \\
\hline
\end{tabular}

for test A, and between $9.09-10.36 \mathrm{~mm}$, instead of nominal $10 \mathrm{~mm}$ and the average of $9.77 \mathrm{~mm}$ which correspond to a disposition of $0,23 \mathrm{~mm}$ for test B.

In most articles which assessing the leaf position accuracy with the garden fence test, 11 thin bands with $2 \mathrm{~mm}$ width have been used. However, we have performed this test with 5 thin bands (Figure 2) due to our field size and over-traveling limitation [24, 25]. As we can see in Table 1, All the values for Garden Fence test were considered to be within a safe $2 \mathrm{~mm}$ limit based on AAPM Reports Task Group 142 [26]. Christos antypas [24] also reported 0.1$0.5 \mathrm{~mm}$ disposition for Siemens Oncor with OPTIFOCUS MLC with the garden fence test which is so close to our results in this study. Picket fence is another similar test that assessing the accuracy of the MLC leaf position, it is like the garden fence test, but the Radiation areas are $5 \mathrm{Cm}$ with no intervals. Pipspro
(Standard Imaging, Inc, USA) and Artiscan imadose (Aquilab, France) are the new softwares which can assess the leaf position accuracy automatically.

\section{B. Leaf Leakage}

When jaws were widely open and MLC Leaves were closed, the Average and Maximum radiation transmission of the MLC leaves were noted $1.79 \%$ and $4.1 \%$ of the open $10 \times 10 \mathrm{Cm}^{2}$ radiation-field at isocenter for 6 MV beams and $1.98 \%$ and $4.4 \%$ for $18 \mathrm{MV}$ beams, respectively (A Direction in Figure 3). The average leaf transmission at the end of the leaves in a closed position (B direction in Figure 3) was noted $18.8 \%$ and $19.5 \%$ for $6 \mathrm{MV}$ and $18 \mathrm{MV}$ beams, respectively. The average and maximum leaf transmission in an interdigit position were also noted $2.5 \%$ and $3.5 \%$ for $6 \mathrm{MV}$ beams and $2.6 \%$ and $3.7 \%$ for $18 \mathrm{MV}$ beams, respectively.

All the transmission measurements were in agreement with acceptance levels based on IPEM 94 [27] and AAPM TG-142 [26] Protocols. Due to the rounded leaf end design, the most transmission was calculated below the leaf end positions. Carlos Daniel Venencia et al. [17] and Thomas Losasso et al. [28] measured the leaf leakage of 120-leaf MLC model Millennium of Varian Clinac21EX 1.5\% for 6 MV Beam. Jun Li and et al. [29] have mea-

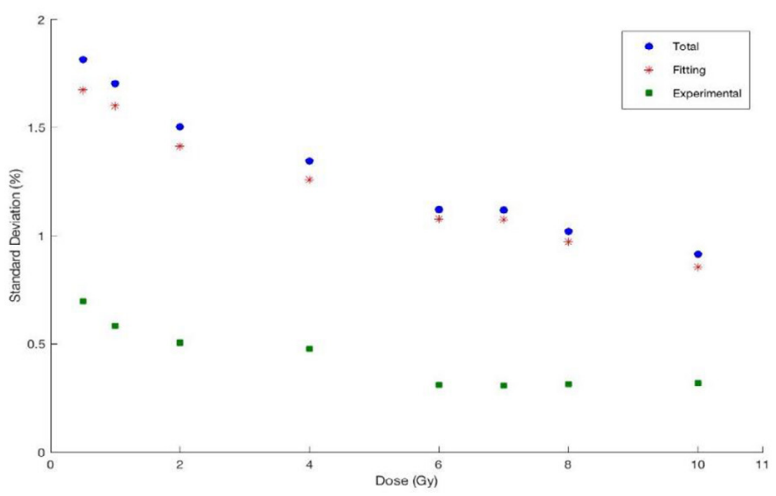

Figure 4: Standard deviation for different delivered dose to EBT3 film. 
sured the average MLC transmission of ELEKTA model Synergy-s about $2 \%$ and the leaf end transmission rate from $25 \%$ to $30 \%$ for $6 \mathrm{MV}, 10 \mathrm{MV}$ and $18 \mathrm{MV}$ beams.

Parameters $n, b$, c in equation 3 were calculated 2, -783.54 and 16297.25, respectively. The total dose uncertainties were between 1-2 $\%$. As shown in Figure 4, the fitting uncertainties were more than the experiment uncertainties for all doses and also the dose uncertainties were reduced by increasing the dose. In TRS-398 document, the dose uncertainty must be within $2 \%$ when the protocol is accurately accomplished [23].

Since this MLC was retrofitted to the Varian CLINAC $2100 \mathrm{C} / \mathrm{D}$ linear accelerator as an add-on one, and was not replaced with conventional jaws, the clearance from the isocenter was reduced. before such a system can be clinically used, beam characteristics and mechanical aspects should be verified [30]. Leaf transmission, the leaf position accuracy and deviations of leaf position from planned position were evaluated in this study and more investigations like changes in scatter factors, percentage depth doses, off-axis profiles must be evaluated in future studies.

\section{Conclusion}

The complete set of the mechanical and dosimetric tests must be done before clinically use of Add-On MLCs. In this study, application of gafchromic EBT3 films for quality assurance of Euromechanics multileaf collimator was studied. This study showed that the average leaf leakage and positional accuracy of this type of MLC were under than the acceptance level based on the protocols.

\section{Acknowledgment}

The current study was supported by a grant from Tehran University of Medical Sciences, Tehran, Iran coded 95-01-30-31669.

\section{Conflict of Interest}

None
References

1. Graves MN, Thompson AV, Martel MK, McShan DL, Fraass BA. Calibration and quality assurance for rounded leaf-end MLC systems. Med Phys. 2001;28:2227-33. doi: 10.1118/1.1413517. PubMed PMID: 11764026.

2. Deng J, Pawlicki T, Chen $Y$, Li J, Jiang SB, Ma CM. The MLC tongue-and-groove effect on IMRT dose distributions. Phys Med Biol. 2001;46:1039-60. PubMed PMID: 11324950.

3. Sharpe MB, Miller BM, Wong JW. Compensation of $\mathrm{x}$-ray beam penumbra in conformal radiotherapy. Med Phys. 2000;27:1739-45. doi: 10.1118/1.1287283. PubMed PMID: 10984219.

4. Yang $Y$, Xing $L$. Using the volumetric effect of a finite-sized detector for routine quality assurance of multileaf collimator leaf positioning. Med Phys. 2003;30:433-41. doi: 10.1118/1.1543150. PubMed PMID: 12674244.

5. Bayouth JE, Wendt D, Morrill SM. MLC quality assurance techniques for IMRT applications. Med Phys. 2003;30:743-50. doi: 10.1118/1.1564091. PubMed PMID: 12772980.

6. LoSasso T, Kutcher GJ. Multileaf collimation versus alloy blocks: analysis of geometric accuracy. Int J Radiat Oncol Biol Phys. 1995;32:499-506. doi: 10.1016/0360-3016(94)00455-t . PubMed PMID: 7751191.

7. LoSasso T, Chui CS, Kutcher GJ, Leibel SA, Fuks $Z$, Ling CC. The use of a multi-leaf collimator for conformal radiotherapy of carcinomas of the prostate and nasopharynx. Int J Radiat Oncol Biol Phys. 1993;25:161-70. doi: 10.1016/03603016(93)90337-u .PubMed PMID: 8420865.

8. Galvin JM, Smith AR, Lally B. Characterization of a multi-leaf collimator system. Int J Radiat Oncol Biol Phys. 1993;25:181-92doi: 10.1016/03603016(93)90339-w. PubMed PMID: 8420867.

9. Wang L, Li J, Paskalev K, Hoban P, Luo W, Chen $L$, et al. Commissioning and quality assurance of a commercial stereotactic treatmentplanning system for extracranial IMRT. J Appl Clin Med Phys. 2006;7:21-34.doi: 10.1120/ jacmp.2027.25368 . PubMed PMID: 16518314; PubMed Central PMCID: PMC5722476.

10. Belec J, Patrocinio H, Verhaegen F. Development of a Monte Carlo model for the Brainlab microMLC. Phys Med Biol. 2005;50:787-99. doi: 10.1088/0031-9155/50/5/005. PubMed PMID: 15798255.

11. Casanova Borca V, Pasquino M, Russo G, 
Grosso P, Cante D, Sciacero P, et al. Dosimetric characterization and use of GAFCHROMIC EBT3 film for IMRT dose verification. J App/ Clin Med Phys. 2013;14:4111. doi: 10.1120/jacmp. v14i2.4111. PubMed PMID: 23470940; PubMed Central PMCID: PMC5714357.

12. Farah N, Francis Z, Abboud M. Analysis of the EBT3 Gafchromic film irradiated with 6 MV photons and $6 \mathrm{MeV}$ electrons using reflective mode scanners. Phys Med. 2014;30:708-12. doi: 10.1016/j.ejmp.2014.04.010. PubMed PMID: 24880678.

13. International Specialty Products (ISP) [Internet]. Gafchromic $^{T M}$ Dosimetry Media, Type EBT-3. Avilale from: http://www.gafchromic.com/documents/EBT3_Specifications.pdf

14. Niroomand-Rad A, Blackwell CR, Coursey BM, Gall KP, Galvin JM, McLaughlin WL, et al. Radiochromic film dosimetry: recommendations of AAPM Radiation Therapy Committee Task Group 55. American Association of Physicists in Medicine. Med Phys. 1998;25:2093-115. doi: 10.1118/1.598407. PubMed PMID: 9829234.

15. Chui CS, Spirou S, LoSasso T. Testing of dynamic multileaf collimation. Med Phys. 1996;23:63541. doi: 10.1118/1.597699. PubMed PMID: 8724734.

16. Bhardwaj AK, Kehwar TS, Chakarvarti SK, Oinam AS, Sharma SC. Dosimetric and qualitative analysis of kinetic properties of millennium 80 multileaf collimator system for dynamic intensity modulated radiotherapy treatments. J Cancer Res Ther. 2007;3:23-8.doi: 10.4103/09731482.31967. PubMed PMID: 17998715.

17. Venencia CD, Besa P. Commissioning and quality assurance for intensity modulated radiotherapy with dynamic multileaf collimator: experience of the Pontificia Universidad Catolica de Chile. $J$ Appl Clin Med Phys. 2004;5:37-54.doi: 10.1120/ jacmp.2021.25275 . PubMed PMID: 15753938; PubMed Central PMCID: PMC5723486.

18. Mamalui-Hunter M, Li H, Low DA. MLC quality assurance using EPID: a fitting technique with subpixel precision. Med Phys. 2008;35:234755. doi: 10.1118/1.2919560. PubMed PMID: 18649468.

19. Chang J, Obcemea CH, Sillanpaa J, Mechalakos J, Burman C. Use of EPID for leaf position accuracy $Q A$ of dynamic multi-leaf collimator (DMLC) treatment. Med Phys. 2004;31:2091-6. doi: 10.1118/1.1760187. PubMed PMID: 15305462.
20. Baghani HR, Aghamiri SM, Mahdavi SR, Robatjazi M, Zadeh AR, Akbari ME, et al. Dosimetric evaluation of Gafchromic EBT2 film for breast intraoperative electron radiotherapy verification. Phys Med. 2015;31:37-42. doi: 10.1016/j. ejmp.2014.08.005. PubMed PMID: 25231546.

21. Falahati F, Nickfarjam A, Shabani M. A feasibility study of IMRT of lung cancer using gafchromic EBT3 film. Journal of Biomedical Physics and Engineering. 2018;8.doi: 10.31661/jbpe. v0i0.791.

22. Devic S, Seuntjens J, Sham E, Podgorsak EB, Schmidtlein CR, Kirov AS, et al. Precise radiochromic film dosimetry using a flat-bed document scanner. Med Phys. 2005;32:2245-53. doi: 10.1118/1.1929253. PubMed PMID: 16121579.

23. Sorriaux J, Kacperek A, Rossomme $S$, Lee $J A$, Bertrand D, Vynckier S, et al. Evaluation of Gafchromic ${ }^{\circledR}$ EBT3 films characteristics in therapy photon, electron and proton beams. Phys Med. 2013;29:599-606.doi: 10.1016/j. ejmp.2012.10.001.

24. Antypas C, Floros I, Rouchota M, Armpilia C, Lyra M. MLC positional accuracy evaluation through the Picket Fence test on EBT2 films and a 3D volumetric phantom. J Appl Clin Med Phys. 2015;16:5185. doi: 10.1120/jacmp.v16i2.5185. PubMed PMID: 26103188; PubMed Central PMCID: PMC5690090.

25. Sumida I, Yamaguchi H, Kizaki H, Koizumi M, Ogata T, Takahashi Y, et al. Quality assurance of MLC leaf position accuracy and relative dose effect at the MLC abutment region using an electronic portal imaging device. $J$ Radiat Res. 2012;53:798-806. doi: 10.1093/jrr/rrs038. PubMed PMID: 22843372; PubMed Central PMCID: PMC3430416.

26. Klein EE, Hanley J, Bayouth J, Yin FF, Simon W, Dresser S, et al. Task Group 142 report: quality assurance of medical accelerators. Med Phys. 2009;36:4197-212. doi: 10.1118/1.3190392. PubMed PMID: 19810494.

27. Kirby M, Ryde S, Hall C. Acceptance testing and commissioning of linear accelerators. York, UK: Institute of Physics and Engineering in Medicine. 2006.

28. Losasso T. IMRT delivery performance with a varian multileaf collimator. Int $J$ Radiat $O n$ col Biol Phys. 2008;71:S85-8. doi: 10.1016/j. ijrobp.2007.06.082. PubMed PMID: 18406945.

29. Li J, Zhang X-Z, Gui L-G, Zhang J, Tang X-B, 
Ge $Y$, et al. Clinical Feasibility of Leakage and Transmission Radiation Dosimetry Using Multileaf Collimator of ELEKTA Synergy-S Accelerator During Conventional Radiotherapy. Journal of Medical Imaging and Health Informatics. 2016;6:409-15.doi: 10.1166/jmihi.2016.1706.
30. Das IJ, Desobry GE, McNeeley SW, Cheng EC, Schultheiss TE. Beam characteristics of a retrofitted double-focused multileaf collimator. Med Phys. 1998;25:1676-84. doi: 10.1118/1.598348. PubMed PMID: 9775373. 